\title{
ORNITHOLOGICAL OBSERVATIONS FROM MARATUA AND BAWEAN ISLANDS, INDONESIA
}

\author{
Ryan C. Burner*1 ${ }^{1}$ Subir B. Shakya ${ }^{1}$, Tri Haryoko ${ }^{2}$, M. Irham $^{2}$, \\ Dewi M. Prawiradilaga ${ }^{2}$ and Frederick H. Sheldon ${ }^{1}$ \\ ${ }^{1}$ Museum of Natural Science and Department of Biological Sciences, \\ Louisiana State University, Baton Rouge, Louisiana, USA \\ ${ }^{2}$ Zoology Division (Museum Zoologicum Bogoriense), Research Center for Biology, \\ Indonesian Institute for Sciences, Jl. Raya Jakarta-Bogor Km. 46 Cibinong, Bogor 16911, Indonesia \\ *Corresponding author: ryan.c.burner@gmail.com
}

Received: 4 January 2018; Accepted: 2 October 2018

\begin{abstract}
Indonesia's many islands, large and small, make it an important center of avian diversity and endemism. Current biogeographic understanding, however, is limited by the lack of modern genetic samples for comparative analyses from most of these islands, and conservation efforts are hampered by the paucity of recent information from small islands peripheral to major, more commonly visited islands. In November and December 2016, we visited Maratua, an oceanic coral atoll $50 \mathrm{~km}$ east of Borneo, and Bawean, a volcanic island on the Sunda continental shelf $150 \mathrm{~km}$ north of Java, to survey birds and collect specimens for morphological and genetic analysis. We detected many of the birds on Maratua's historical lists and added several new resident and migratory species. Notably, we did not detect the Maratua White-rumped Shama (Copsychus malabaricus barbouri). On Bawean, we found the forests to be nearly silent and detected remarkably few resident land-bird species overall. The severe population reduction of C. m. barbouri on Maratua and the drastic reduction of forest birds on Bawean probably result from overexploitation by the cage-bird trade in the first case and a combination of the cage-bird trade and pellet-gun hunting in the second.
\end{abstract}

Keywords: Avifauna, Borneo, cage-bird trade, extirpation, Sundaland

\section{INTRODUCTION}

The Sunda Islands of Indonesia constitute an important centre of avian diversity and endemism (Kier et al., 2009; de Bruyn et al., 2014; Sheldon et al., 2015). However, many holes remain in our understanding of the relationships and dynamics of bird populations on these islands due to a lack of recent ornithological surveys and modern biological specimens (Sheldon et al., 2009; Eaton et al., 2016; Manthey et al., 2017). Of particular interest are the many small islands adjacent to the Greater Sunda Islands. These small islands, especially if they are oceanic, may act as refugia, sheltering their native residents from ecological forces affecting populations on adjacent larger islands (Fooden, 1995; Chua et al., 2015). As such, they may act as museums or natural experiments in biogeography, potentially providing insight into the evolution of Sundaic biodiversity as a whole. There is, however, a paucity of recent information on the avifaunas of these small islands, and changes in them are expected due to the increasing human population pressure and especially the region's flourishing cage-bird trade (Eaton et al., 2015; Shepherd et al., 2016; Bergin et al., 2017).

One such island, with a rich ornithological history, is Maratua (Fig. 1), a $24 \mathrm{~km}^{2}$ coral atoll that is part of the Derawan Islands located $50 \mathrm{~km}$ east of Berau Regency, East Kalimantan, Indonesian Borneo. Most of what we know about Maratua's avifauna comes 


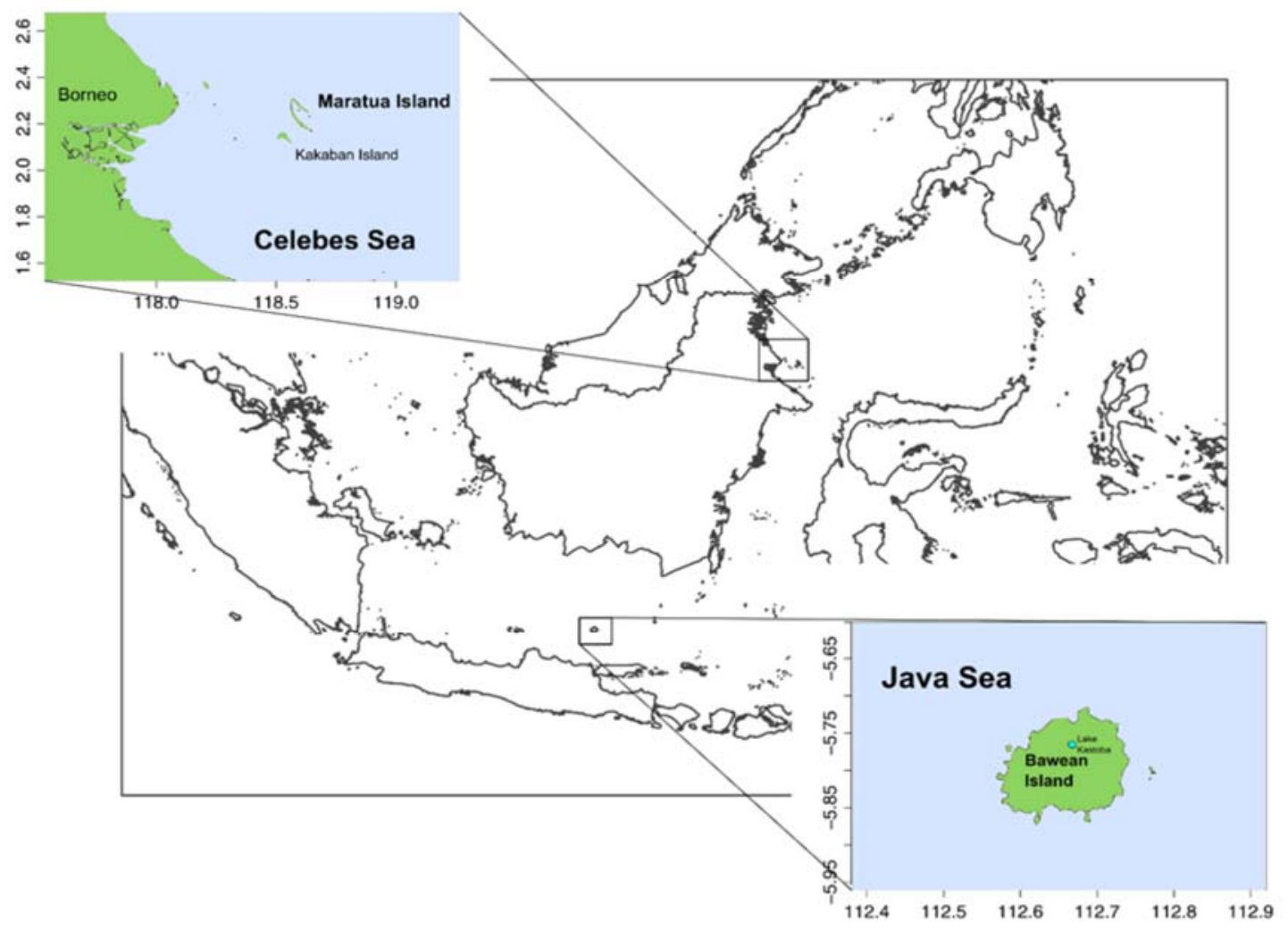

Figure 1. Location of Maratua and Bawean Islands, Indonesia.

from expeditions in the early $20^{\text {th }}$ Century by H. C. Raven and E. P. Mjöberg (Bangs \& Peters, 1927; Riley, 1930). Combined, these expeditions collected or observed 24 species on Maratua (Table 1). Twenty of these species are apparent resident land-birds, while the remaining four are water birds and migrants. After these original expeditions, the island's avifauna remained largely unexamined for nearly 100 years until the short surveys of Q. Phillipps in 2009, 2010, and 2011 (pers. comm., Phillipps \& Phillipps, 2014; Chua et al., 2015). For most of the last century, published works on Maratua's natural history emphasized its abundant marine life rather than its land animals ( $\mathrm{Ng} \&$ Tomascik, 1994; de Voogd et al., 2009; Becking et al., 2011).

Maratua is biogeographically interesting because it is oceanic and permanently separated from Borneo and the Sunda continental shelf by a channel more than $180 \mathrm{~m}$ deep. The island has never been connected by land to mainland Borneo, even during the dramatically low sea-levels of Pleistocene glacial maxima, which joined all islands on the Sunda continental shelf into a single land mass (Voris, 2000). One result of its permanent isolation (and size) is that Maratua's species richness is much lower than on adjacent Borneo, causing the few species that occur there to be more abundant than usual, a common island phenomenon (MacArthur et al., 1972). Another result is that several bird populations on Maratua 
are phenotypically distinct from populations on adjacent Borneo. These distinct taxa were described as separate species following their discovery (Bangs \& Peters, 1927; Riley, 1930), but most are now treated as subspecies of more widespread, polytypic species: e.g., Blackheaded Bulbul Pycnonotus atriceps hodiernus, White-rumped Shama Copsychus malabaricus barbouri, and Black-naped Monarch Hypothymis azurea aeria (Clements et al., 2017). These three taxa were the subject of a recent study which used DNA extracted from toepads of the original specimens to examine their relationships to populations on Borneo and elsewhere in Southeast Asia (Chua et al., 2015). That study found that P. a. hodiernus and C. m. barbouri are strongly differentiated from their sister taxa on Borneo, but $H$. a. aeria varies only slightly from $H$. azurea elsewhere in Southeast Asia, implying either continued gene flow between $H$. azurea populations on Maratua and Borneo or recent colonization of the smaller island (Chua et al., 2015).

Another small island of particular ornithological interest is Bawean $\left(210 \mathrm{~km}^{2}\right)$, which lies $150 \mathrm{~km}$ north of Surabaya, Java, in the Javan Sea (Fig. 1). Unlike Maratua, Bawean is a volcanic island and lies on the Sunda continental shelf. Thus, it was connected periodically to Java and Borneo during Pleistocene glacial events, and its bird populations would have experienced periodic gene flow from the larger islands. The first museum collectors to visit Bawean were A. G. Vorderman in 1891-1892 (Vorderman, 1892) and W. L. Abbott in 1907 (Oberholser, 1917). Combined, these two expeditions found 26 species on the island, and taxonomic comparisons indicated that most of the species were similar to respective taxa on Java. Later, Hoogerwerf $(1962,1965,1966,1967)$ expanded the list to 52 species (Table 2). As with Maratua's populations, many Bawean taxa were originally described as endemic species or subspecies (nine in all). Nowadays, most are recognized as subspecies, e.g., Crested Serpent-Eagle Spilornis cheela baweanus, Black-headed Bulbul Pycnonotus atriceps baweanus, Ashy Tailorbird Orthotomus ruficeps baweanus, and Abbott's Babbler Turdinus abbotti baweanus (Clements et al., 2017).

Ornithologists from the Museum Zoologicum Bogoriense, Indonesian Institute of Sciences (MZB), and Louisiana State University Museum of Natural Science (LSUMNS) visited Maratua Island from 18-23 November 2016 and Bawean from 1-5 December 2016. The aim of these expeditions was to do inventory work of the avifauna of both islands and collect specimens for molecular biogeographic comparisons of populations across Sundaland (Sheldon et al., 2015; Lim et al., 2017; Shakya \& Sheldon, 2017; Shakya et al., 2018). Obtaining updated information on the avifauna of these and other Sundaic islands is especially important given the prevalence of the cage-bird trade and forest loss throughout Indonesia, which has resulted in the near extinction of some species and extirpation of several taxa from locations throughout the region (Jepson \& Ladle, 2005; Wilcove et al., 2013; Eaton et al., 2015; Bergin et al., 2017). 


\section{MATERIALS AND METHODS}

\section{Maratua Expedition}

Our Maratua field team members included a researcher from MZB (TH) and two researchers from LSUMNS (RCB, SBS). We flew from Samarinda, East Kalimantan, to Kalimarau Airport, East Kalimantan, and drove to Tanjung Batu where we boarded a speedboat for Maratua Island. We stayed at Maratua Guesthouse $\left(2.267^{\circ} \mathrm{N}, 118.563^{\circ} \mathrm{E}\right)$ and set ten mist nets in the low but dense forest within $200 \mathrm{~m}$ of the guesthouse (Fig. 2), as well as along trails and openings. Nets were set from sunrise to sunset for five days November 19 $-22^{\text {nd }}, 2016$. Most of our effort was spent tending nets and preparing specimens, but we opportunistically noted birds seen or heard near the guesthouse while tending nets and during about 8 person-hours of birding within $1 \mathrm{~km}$ of the site. We were not able to explore the rest of this atoll.

\section{Bawean Expedition}

The Bawean expedition consisted of one researcher each from MZB (M. Irham) and LSUMNS (SBS). A field technician (Suparno) from MZB also participated. We took a boat from Gresik, north of the city of Surabaya in eastern Java, to Sangkapura on Bawean Island. We stayed at the home of the local forestry officer and set mist nets nearby on a forested mountain-side (Fig. 2; 5.816 $\mathrm{S}, 112.632^{\circ} \mathrm{E}$ ). As with Maratua, we periodically walked the trails and noted species seen and heard.

\section{RESULTS}

\section{Maratua Expedition}

We detected a total of 25 species on Maratua (Table 1) and collected 12. Of these, 14 were apparent resident land-birds, five were transient water birds or raptors, and six were long-distance migrants. Of the resident land-birds, three are additions to the early $20^{\text {th }}$ century lists: Asian Emerald Dove Chalcophaps indica, Black-naped Fruit-Dove Ptilinopus melanospilus and Crimson Sunbird Aethopyga siparaja. The two doves were previously detected by Q. Phillipps (unpublished report); A. siparaja represents a new record for Maratua.

In addition to these new residents, we also collected specimens of four migratory species--Arctic Warbler Phylloscopus borealis, Gray-streaked Flycatcher Muscicapa griseisticta, Siberian Blue Robin Larvivora cyane, and Narcissus Flycatcher Ficedula narcissina--and photographed a fift--Mugimaki Flycatcher (Ficedula mugimaki). All five represent new records for the Maratua, and the Gray-streaked Flycatcher is likely the first specimen from Borneo. Noticeably absent from our observations were Pied Triller (Lalage nigra) and White-rumped Shama (Copsychus malabaricus). On a brief stop at nearby Kakaban Island we recorded swiftlets (Aerodramus sp.), Pacific Reef-Heron (Egretta sacra), and Pacific swallow (Hirundo tahitica), but did not survey the forest. Details on Maratua species of special interest are provided below. 

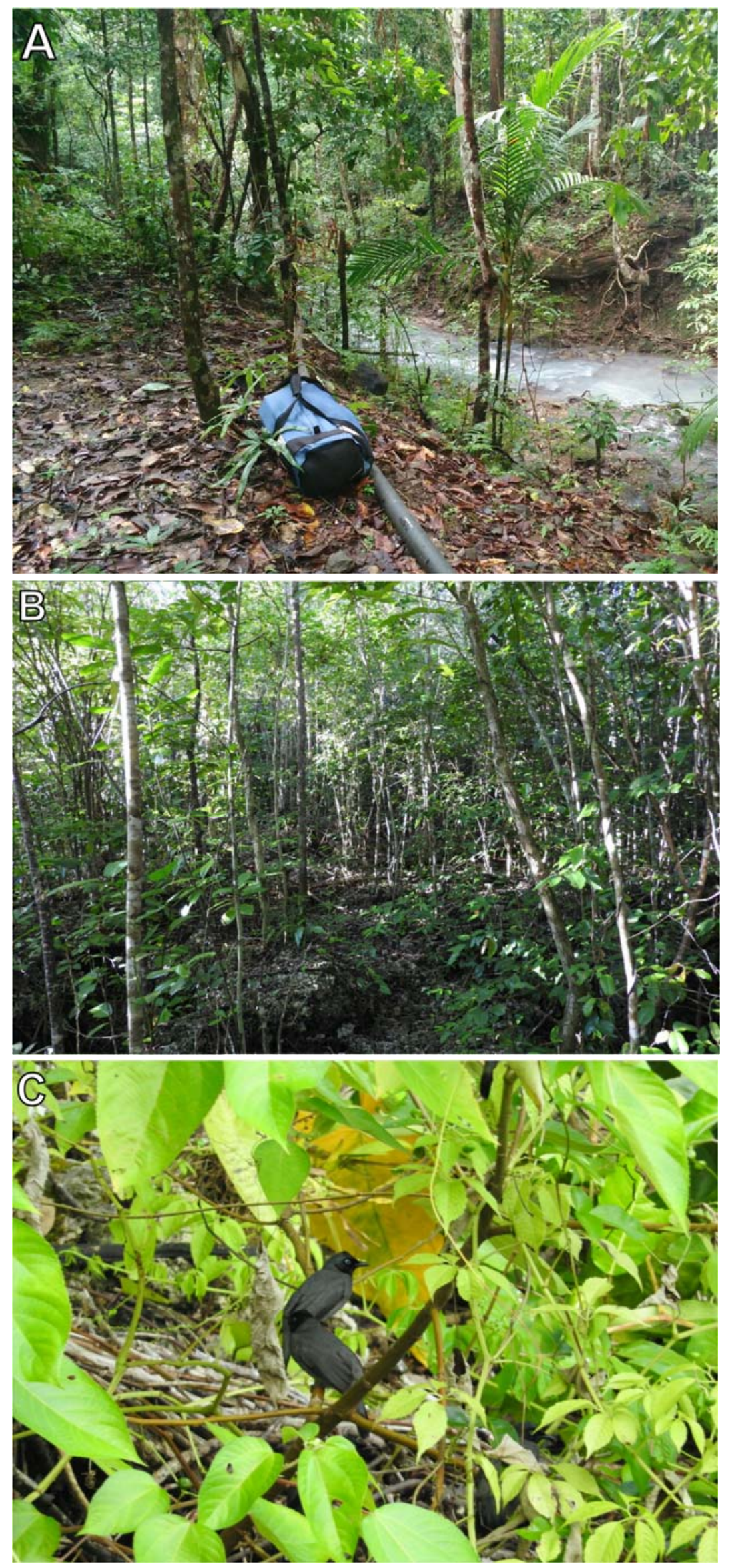

Figure 2. Typical habitat in A) Bawean Island and B) Maratua Island. C) A pair of Black-headed Bulbuls (Pycnonotus atriceps hodiernus) from Maratua Island. 
Tabon Scrubfowl Megapodius cumingii: We heard and recorded calls from one individual on several occasions from deep within the forest across the lagoon from the Maratua guesthouse (for audio recording, see eBird checklist S40957930). This species occurs widely in Sulawesi and the Philippines, and islands off northeast Borneo. Maratua is its southernmost Bornean locality.

Metallic Pigeon Columba vitiensis: We did not detect this bird, nor was it collected during early Maratua expeditions, but Q. Phillipps (unpublished report) identified one in a 2010 photo taken by a local person.

Black-naped Fruit-Dove: A new record for Maratua (also detected by Q. Phillipps, unpublished report), although one was reported by Riley (1930) from Pulau Panjang nearer the mainland.

Northern Boobook (Ninox japonica): Boreal migrant. We did not detect this species, nor was it collected during the early Maratua expeditions, but Q. Phillipps (unpublished report) saw this migratory species on both Maratua and Kakaban in 2011.

Pied Triller: Although conspicuously absent during our trip, multiple specimens were collected on early expeditions to Maratua (Bangs \& Peters, 1927; Riley, 1930). Q. Phillipps (unpublished report) found them to be uncommon in 2009-2010. We did not focus much effort on beach-strand habitat and, thus, could have missed this species.

Arctic Warbler: Boreal migrant. We found this species to be common but were not able to distinguish between subspecies recognized by Clements et al. (2017). Some authorities now consider these subspecies as three distinct species (Eaton et al., 2016). Gray-streaked Flycatcher: We netted one individual, which is probably the first specimen (Fig. 3) from anywhere in Borneo. It was identified based on its heavy, distinct ventral streaking and very long primary projection (David Wells, pers. comm.,14 August 2018). This Boreal migrant typically winters from the Philippines to New Guinea.

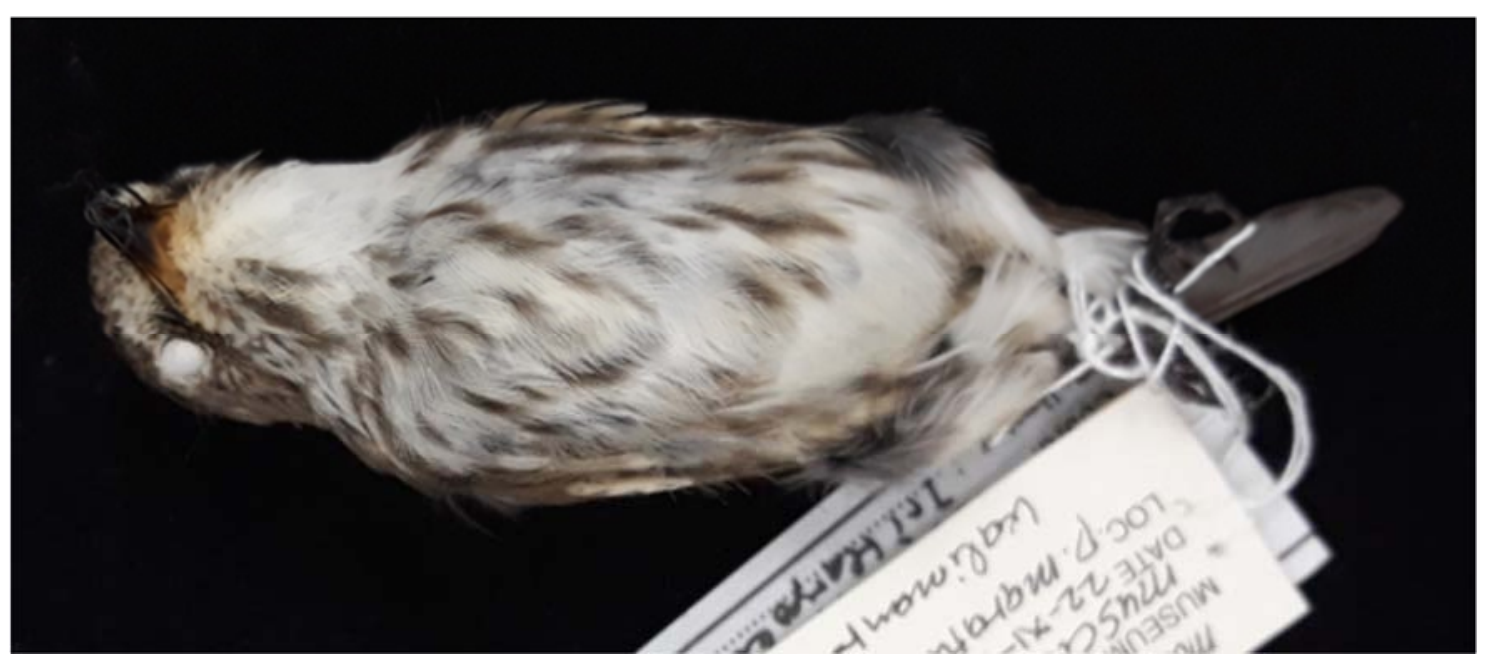

Figure 3. Likely the fir st specimen of Gray-streaked Flycatcher (Muscicapa griseisticta) from Borneo, netted on Maratua Island in November 2017. The bold and distinct vertical streaking on the breast and long primary projection are diagnostic for this species. 
White-rumped Shama: This normally easily detected species was notably absent from our Maratua list. We failed to see or hear a single individual. Several were collected during the early expeditions (Bangs \& Peters, 1927; Riley, 1930), and Q. Phillipps (pers. comm.) found the species to be reasonably common in 2010. However, by 2011 the numbers had been drastically reduced, probably as a result of trapping for the cage-bird industry. However, we surveyed a relatively small segment of the island and it is possible that the species occurs elsewhere. More effort is needed to confirm the status of this endemic taxon.

Siberian Blue Robin: Boreal migrant. We netted one individual.

Narcissus Flycatcher: Boreal migrant. This species was fairly common.

Mugimaki Flycatcher: Boreal migrant. We sighted and photographed this species only once (for photograph, see eBird checklist S40957930).

Crimson Sunbird: We recorded a single individual of this species.

Table 1. Bird species recorded on Maratua Island, East Kalimantan, Indonesian Borneo

\begin{tabular}{|c|c|c|c|c|}
\hline Scientific Name ${ }^{a}$ & English Name & $\begin{array}{c}\text { Early } \\
1900 ' s\end{array}$ & $\begin{array}{c}\text { Phillipps } \\
\text { 2009-11 } \\
\end{array}$ & $\begin{array}{c}\text { Expedition } \\
2016^{c}\end{array}$ \\
\hline Megapodius cumingii & Tabon Scrubfowl & $\mathrm{X}$ & $\mathrm{X}$ & $\mathrm{X}$ \\
\hline Fregata ariel & Lesser Frigatebird & & $\mathrm{X}$ & $\mathrm{X}$ \\
\hline Ardea sumatrana & Great-billed Heron & & $\mathrm{X}$ & \\
\hline Egretta garzetta & Little Egret & & $\mathrm{X}$ & \\
\hline Egretta sacra & Pacific Reef-Heron & $\mathrm{X}$ & $\mathrm{X}$ & $\mathrm{X}$ \\
\hline Bubulcus ibis & Cattle Egret & & $\mathrm{X}$ & \\
\hline Butorides striata & Striated Heron & $\mathrm{X}$ & & $\mathrm{X}$ \\
\hline Pandion haliaetus & Osprey & & $\mathrm{X}$ & $\mathrm{X}$ \\
\hline Haliastur indus & Brahminy Kite & & $\mathrm{X}$ & $\mathrm{X}$ \\
\hline Haliaeetus leucogaster & White-bellied Sea-Eagle & & $\mathrm{X}$ & \\
\hline Numenius phaeopus* & Whimbrel & $\mathrm{X}$ & & \\
\hline Actitis hypoleucos* & Common Sandpiper & $\mathrm{X}$ & $\mathrm{X}$ & $\mathrm{X}$ \\
\hline Columba vitiensis & Metallic Pigeon & & $\mathrm{X}^{* *}$ & \\
\hline Chalcophaps indica & Asian Emerald Dove & & $\mathrm{X}$ & S \\
\hline Caloenas nicobarica & Nicobar Pigeon & $\mathrm{X}$ & $\mathrm{X}$ & \\
\hline Treron vernans & Pink-necked Pigeon & $\mathrm{X}$ & $\mathrm{X}$ & $\mathrm{X}$ \\
\hline Ptilinopus melanospilus & Black-naped Fruit-Dove & & $\mathrm{X}$ & $\mathrm{S}$ \\
\hline Ducula pickeringii & Gray Imperial-Pigeon & $\mathrm{X}$ & $\mathrm{X}$ & $\mathrm{X}$ \\
\hline Ducula bicolor & Pied Imperial-pigeon & $\mathrm{X}$ & $\mathrm{X}$ & \\
\hline Ninox japonica* & Northern Boobook & & $\mathrm{X}$ & \\
\hline Aerodramus salangana & Mossy-nest Swiftlet & $\mathrm{X}$ & $\mathrm{X}$ & \\
\hline Aerodramus fuciphagus & White-nest Swiftlet & $\mathrm{X}$ & $\mathrm{X}$ & \\
\hline Alcedo atthis* & Common Kingfisher & & $\mathrm{X}$ & \\
\hline Todiramphus chloris & Collared Kingfisher & $\mathrm{X}$ & $\mathrm{X}$ & $\mathrm{X}$ \\
\hline Tanygnathus lucionensis & Blue-naped Parrot & $\mathrm{X}$ & $\mathrm{X}$ & $\mathrm{X}$ \\
\hline Artamus leucorynchus & White-breasted Woodswallow & $\mathrm{X}$ & & \\
\hline Lalage nigra & Pied Triller & $\mathrm{X}$ & $\mathrm{X}$ & \\
\hline Pachycephala cinerea & Mangrove Whistler & $\mathrm{X}$ & $\mathrm{X}$ & $\mathrm{S}$ \\
\hline Dicrurus hottentottus & Hair-crested Drongo & $\mathrm{X}$ & $\mathrm{X}$ & $\mathrm{S}$ \\
\hline
\end{tabular}


continued ...

\begin{tabular}{|c|c|c|c|c|}
\hline Scientific Name $^{a}$ & English Name & $\begin{array}{c}\text { Early } \\
1900 \text { 's }\end{array}$ & $\begin{array}{c}\text { Phillipps } \\
\text { 2009-11 }\end{array}$ & $\begin{array}{c}\text { Expedition } \\
2016^{c}\end{array}$ \\
\hline Hypothymis azurea & Black-naped Monarch & $\mathrm{X}$ & $\mathrm{X}$ & $\mathrm{S}$ \\
\hline Pycnonotus atriceps & Black-headed bulbul & $\mathrm{X}$ & $\mathrm{X}$ & $\mathrm{S}$ \\
\hline Phylloscopus borealis & Arctic Warbler & & & $\mathrm{S}$ \\
\hline Muscicapa griseisticta* & Gray-streaked Flycatcher & & & $\mathrm{S}$ \\
\hline Copsychus malabaricus & White-rumped Shama & $\mathrm{X}$ & $\mathrm{X}$ & \\
\hline Larvivora cyane* & Siberian Blue Robin & & & $\mathrm{S}$ \\
\hline Ficedula narcissina* & Narcissus Flycatcher & & & $\mathrm{S}$ \\
\hline Ficedula mugimaki* & Mugimaki Flycatcher & & & $\mathrm{X}$ \\
\hline Aplonis panayensis & Asian Glossy Starling & $\mathrm{X}$ & $\mathrm{X}$ & $\mathrm{X}$ \\
\hline Anthreptes malacensis & Plain-throated Sunbird & $\mathrm{X}$ & $\mathrm{X}$ & S \\
\hline Leptocoma brasiliana & Van Hasselt's Sunbird & $\mathrm{X}$ & $\mathrm{X}$ & \\
\hline Leptocoma calcostetha & Copper-throated Sunbird & $\mathrm{X}$ & $\mathrm{X}$ & \\
\hline Aethopyga siparaja & Crimson Sunbird & & & S \\
\hline Totals: & 42 & 24 & 33 & 25 \\
\hline \multicolumn{5}{|c|}{$\begin{array}{l}{ }^{a} \text { Classification follows Clements et al. (2017). } \\
{ }^{b} \text { Lists from Bangs \& Peters (1927) and Riley (1930) } \\
{ }^{c} \mathrm{~S}=\text { specimen records from 2016, X= other records } \\
\text { *Boreal migrant } \\
\text { ** Phillipps reports seeing a photo taken by a local resident in } 2010 \text { (unpublished report) }\end{array}$} \\
\hline
\end{tabular}

\section{Bawean Expedition}

We recorded only 13 and collected only three species on Bawean Island (Table 2). Although the island was well-forested and had many trees in fruit, we saw and heard few birds during our stay. There was, however, an abundance of flying foxes (Pteropus alecto) as well as Cave Swiftlets (Collocalia linchi). Around the villages, we often saw and heard Ashy Tailorbird (Orthotomus ruficeps) and Plain-throated Sunbird (Anthreptes malacensis). In open areas we saw Collared Kingfisher (Todiramphus chloris). Oriental Honey Buzzards (Pernis ptilorhynchus) and Crested Serpent-eagles (Spilornis cheela) were often observed flying over the mountaintops. However, in the forest we had little success in finding birds. We heard Abbott's Babbler (Turdinus abbotti) and were able to use playback to attract this species for visual identification. The most frequently netted species was Rufous-backed Kingfisher (Ceyx rufidorsa). SBS travelled to Lake Kastoba, but was unable to see or hear any birds besides $O$. ruficeps. Black-headed Bulbul (Pycnonotus atriceps) was nowhere to be seen or heard. Based on interviews with the locals, it was apparently the most common forest bird on the island until five years ago. We did manage to obtain one cage-bird of this species, which displayed the grey colour morph, matching those previously described as the dominant form on the island (Hoogerwerf, 1967; Dickinson \& Dekker, 2002). 
Burner et al.: Ornithological observations from Maratua and Bawean ....

Table 2. Bird species recorded on Bawean Island, Java Sea, Indonesia

\begin{tabular}{|c|c|c|c|c|}
\hline Scientific Name ${ }^{a}$ & English Name & $\begin{array}{l}\text { Expeditions } \\
1892,1917^{\mathrm{b}}\end{array}$ & $\begin{array}{c}\text { Hoogerwerf } \\
1966\end{array}$ & $\begin{array}{l}\text { Expedition } \\
\mathbf{2 0 1 6}^{\mathbf{c}}\end{array}$ \\
\hline Anas gibberifrons & Sunda Teal & & $\mathrm{X}$ & \\
\hline Gallus varius & Green Junglefowl & $\mathrm{X}$ & $\mathrm{X}$ & \\
\hline Fregata minor & Great Frigatebird & $\mathrm{X}$ & $\mathrm{X}$ & \\
\hline Ardea sumatrana & Great-billed Heron & $\mathrm{X}$ & $\mathrm{X}$ & \\
\hline Ardea purpurea & Purple Heron & & $\mathrm{X}$ & \\
\hline Ardea alba & Great Egret & & $\mathrm{X}$ & \\
\hline Egretta garzetta & Little Egret & & $\mathrm{X}$ & \\
\hline Egretta sacra & Pacific Reef-Heron & & $\mathrm{X}$ & \\
\hline Butorides striata & Striated Heron & $\mathrm{X}$ & $\mathrm{X}$ & \\
\hline Nycticorax nycticorax & Black-crowned Night-Heron & & $\mathrm{X}$ & \\
\hline Pandion haliaetus & Osprey & & $\mathrm{X}$ & \\
\hline Pernis ptilorhynchus & Oriental Honey-buzzard & & & $\mathrm{X}$ \\
\hline Spilornis cheela & Crested Serpent-Eagle & $\mathrm{X}$ & $\mathrm{X}$ & $\mathrm{X}$ \\
\hline Accipiter soloensis* & Chinese Sparrowhawk & & & $* * *$ \\
\hline Accipiter gularis* & Japanese Sparrowhawk & & & $* * *$ \\
\hline Pluvialis squatarola* & Black-bellied Plover & & $\mathrm{X}$ & \\
\hline Pluvialis fulva* & Pacific Golden-Plover & & $\mathrm{X}$ & \\
\hline Charadrius leschenaultii* & Greater Sand-Plover & & $\mathrm{X}$ & \\
\hline Numenius phaeopus* & Whimbrel & & $\mathrm{X}$ & \\
\hline Actitis hypoleucos* & Common Sandpiper & $\mathrm{X}$ & $\mathrm{X}$ & \\
\hline Tringa brevipes* & Gray-tailed Tattler & & $\mathrm{X}$ & \\
\hline Tringa nebularia* & Common Greenshank & & $\mathrm{X}$ & \\
\hline Tringa totanus* & Common Redshank & & $\mathrm{X}$ & \\
\hline Turnix suscitator & Barred Buttonquail & & $\mathrm{X}$ & \\
\hline Thalasseus bergii & Great Crested Tern & & $\mathrm{X}$ & \\
\hline Streptopelia chinensis & Spotted Dove & $\mathrm{X}$ & $\mathrm{X}$ & \\
\hline Chalcophaps indica & Asian Emerald Dove & $\mathrm{X}$ & $\mathrm{X}$ & \\
\hline Geopelia striata & Zebra Dove & $\mathrm{X}$ & $\mathrm{X}$ & \\
\hline Treron vernans & Pink-necked Pigeon & & $\mathrm{X}$ & \\
\hline Ducula aenea & Green Imperial-Pigeon & & $\mathrm{X}$ & \\
\hline Ducula rosacea & Pink-headed Imperial-Pigeon & & $\mathrm{X}$ & \\
\hline Ducula bicolor & Pied Imperial-Pigeon & & $\mathrm{X}$ & \\
\hline Eudynamys scolopaceus & Asian Koel & $\mathrm{X}$ & $\mathrm{X}$ & \\
\hline Chrysococcyx basalis** & Horsfield's Bronze-Cuckoo & & $\mathrm{X}$ & \\
\hline Cuculus optatus* & Oriental Cuckoo & $\mathrm{X}$ & $\mathrm{X}$ & \\
\hline Strix seloputo & Spotted Wood-Owl & $\mathrm{X}$ & $\mathrm{X}$ & \\
\hline Collocalia linchi & Cave Swiftlet & $\mathrm{X}$ & $\mathrm{X}$ & $\mathrm{X}$ \\
\hline Aerodramus fuciphagus & $\begin{array}{l}\text { White-nest Swiftlet } \\
\text { Rufous-backed Dwarf- }\end{array}$ & & $\mathrm{X}$ & \\
\hline Ceyx rufidorsa & Kingfisher & $\mathrm{X}$ & $\mathrm{X}$ & $\mathrm{X}$ \\
\hline Todiramphus sanctus** & Sacred Kingfisher & & $\mathrm{X}$ & \\
\hline Todiramphus chloris & Collared Kingfisher & $\mathrm{X}$ & $\mathrm{X}$ & $\mathrm{X}$ \\
\hline Oriolus chinensis & Black-naped Oriole & & $\mathrm{X}$ & \\
\hline Pycnonotus atriceps & Black-headed Bulbul & $X$ & $\mathrm{X}$ & $\mathrm{S}^{\dagger}$ \\
\hline
\end{tabular}


continued ...

\begin{tabular}{|c|c|c|c|c|}
\hline Scientific Name ${ }^{a}$ & English Name & $\begin{array}{l}\text { Expeditions } \\
1892,1917^{b} \\
\end{array}$ & $\begin{array}{c}\text { Hoogerwerf } \\
1966 \\
\end{array}$ & $\begin{array}{c}\text { Expedition } \\
2016^{\mathrm{c}} \\
\end{array}$ \\
\hline Pycnonotus plumosus & Olive-winged Bulbul & $\mathrm{X}$ & $\mathrm{X}$ & S \\
\hline Phylloscopus borealis* & Arctic Warbler & & $\mathrm{X}$ & S \\
\hline Orthotomus ruficeps & Ashy Tailorbird & $\mathrm{X}$ & $\mathrm{X}$ & $\mathrm{X}$ \\
\hline Turdinus abbotti & Abbott's Babbler & $\mathrm{X}$ & $\mathrm{X}$ & $\mathrm{S}$ \\
\hline Gracula religiosa & Common Hill Myna & $\mathrm{X}$ & $\mathrm{X}$ & \\
\hline Dicaeum trochileum & Scarlet-headed Flowerpecker & $\mathrm{X}$ & $\mathrm{X}$ & $\mathrm{X}$ \\
\hline Anthreptes malacensis & Plain-throated Sunbird & $\mathrm{X}$ & $\mathrm{X}$ & $\mathrm{X}$ \\
\hline Cinnyris jugularis & Olive-backed Sunbird & $\mathrm{X}$ & $\mathrm{X}$ & $\mathrm{X}$ \\
\hline Passer montanus & Eurasian Tree Sparrow & & $\mathrm{X}$ & \\
\hline Ploceus manyar & Streaked Weaver & $\mathrm{X}$ & & \\
\hline Lonchura punctulata & Scaly-breasted Munia & $\mathrm{X}$ & $\mathrm{X}$ & \\
\hline Lonchura oryzivora & Java Sparrow & & $\mathrm{X}$ & \\
\hline Totals: & 55 & 25 & 51 & 13 \\
\hline $\begin{array}{l}{ }^{\mathrm{a}} \text { Classification follows Clen } \\
{ }^{\mathrm{b}} \text { From Vorderman (1892) at } \\
{ }^{\mathrm{c}} \mathrm{S}=\text { specimen records from } \\
{ }^{*} \text { Boreal migrant } \\
{ }^{* *} \text { Austral migrant } \\
{ }^{* * *} \text { Added by Nijman (2004 } \\
\dagger \text { Cage-bird collected, no wil }\end{array}$ & $\begin{array}{l}\text { detected on our } 2016 \text { expedition } \\
\text { duals detected }\end{array}$ & & & \\
\hline
\end{tabular}

\section{DISCUSSION}

\section{Maratua Expedition}

As a permanently isolated oceanic island close enough to the Bornean coast to receive occasional invaders but far enough to prevent much gene flow, Maratua potentially acts as a "museum," preserving the morphology of some early inhabitants of Sundaland (Fooden, 1995; Chua et al., 2015). Periodic surveys of birds there provide snapshots of avifaunal changes on the island through time. The island's birds were well surveyed by Q. Phillipps in the late 2000s (pers. comm.; Phillipps \& Phillipps, 2014; Chua et al., 2015), but even so we recorded additional species and two possible losses to the avifauna.

The expedition recorded 12 land-bird species, of which seven are new for the island: three likely residents--Asian Emerald Dove, Black-naped Fruit-Dove, and Crimson Sunbird; and five Boreal migrants--Arctic Warbler, Gray-streaked Flycatcher, Siberian Blue Robin, Narcissus Flycatcher, and Mugimaki Flycatcher (Table 1). We failed, however, to detect two species previously found on the island: Pied Triller and White-rumped Shama. The triller was never abundant on Maratua, and we may simply have failed to locate one because we focused on inland rather than coastal habitat. The shama was common on Maratua 10 years ago but was reduced in numbers by 2011 (Q. Phillipps, unpublished manuscript, pers. comm.). 
This species is conspicuous when present because of its loud, warbling song, and it is easily mist-netted. Thus, we are confident that it did not occur in the area we worked most intensively (i.e., forest within one $\mathrm{km}$ of the resthouse). The shama may still occur in other parts of the island but has been dramatically reduced (if not extirpated), presumably by pettraders who value it above most other species (Eaton et al., 2015). Its loss would be a great tragedy because the Maratua subspecies, C. m. barbouri, represents an evolutionarily important lineage relative to C. m. malabaricus and C. m. stricklandii on mainland Borneo (Chua et al., 2015). Indeed, some classifications even treat it as a distinct species, $C$. barbouri (Eaton et al., 2016).

\section{Bawean Expedition}

Although relatively distant from Java and Borneo, Bawean Island is, in principle, inhabited or visited by at least 52 species of resident and migrant birds (Table 2), including several endemic land-bird taxa, based on historical surveys in the first half of the $20^{\text {th }}$ Century (Oberholser, 1917; Hoogerwerf, 1966, 1967). Since these surveys, however, little ornithological work has been carried out on the island, except for a 2002 study of owls and birds of prey by Nijman $(2004,2006)$. He added three new species of large predatory birds to the island's list, bringing the historical total to 55, but did not comment on the status of species in other families. Our expedition detected only 13 species on the island, in part because our visit was short and we did not survey coastal areas. However, of great concern was that we found only nine of 25 previously common resident land-birds (excluding migrants, owls, and birds of prey). In short, the silence in the forests and fields of the island was remarkable.

Based on interviews with residents, Nijman (2006) reported that pellet guns became common on the island in the late 1990s, and that bird hunting is common. Additionally, the island is relatively close to Java, where many species are in high demand as cage-birds (Eaton et al., 2015). This combination of hunting pressure and the songbird trade seems likely to have reduced and altered the avifauna of the island significantly. We detected only four of the nine endemic taxa in the wild. The apparent absence of the usually conspicuous Black-headed Bulbul makes it likely that this species has been extirpated from the island. We also failed to detect Barred Buttonquail (Turnix suscitator) and Spotted Wood-Owl (Strix seloputo), but are not sure this reflects their absence on the island. The former can be difficult to detect, while in the case of the latter we did not try to survey for nocturnal birds. Common Hill Myna (Gracula religiosa) and Scaly-breasted Munia (Lonchura punctulata), two other species with endemic Bawean subspecies, are usually easily detected but were not observed on our expedition. We also did not detect any of the seven dove and pigeon species previously known to occur on the island. These are likely to be popular prey of local hunters. 


\section{CONCLUSIONS}

Our expeditions to Maratua and Bawean islands, Indonesia, provide the first modern genetic samples from these locations, as well as updated species lists. They document a significant change in avifaunas on the two islands, as well as new records of migratory and resident species for Maratua. Of the two islands, the current bird community on Maratua is richer and more closely resembles the historic community than does that of Bawean, where extirpation appears significant. Nevertheless, on Maratua it is possible that at least one species (White-rumped Shama) may have been extirpated, though hopefully it still occurs in small numbers. These observations confirm the need for action against the cage-bird trade in Indonesia, which is a significant threat to the survival of several taxa. On small islands with endemic taxa, the genetic effects of local extirpations are irreversible.

\section{ACKNOWLEDGEMENTS}

Indonesia State Ministry of Research and Technology (RISTEK research license numbers 388/SIP/FRP/E5/Dit.KI/XI/2016 and 389/SIP/FRP/E5/Dit.KI/XI/2016) and the Ministry of Forestry granted permission to undertake research on Maratua and Bawean. We also thank the Research Center for Biology, Indonesian Institute of Science (RCB-LIPI), and the Museum Zoologicum Bogoriensis for their invaluable support and Lindsay Burner for help in the field. Quentin Phillipps kindly provided a report of his visits to Maratua and permission to use it. Three anonymous reviewers provided helpful comments on the manuscript. This work was supported by the U.S. National Science Foundation grant 1241059 and Louisiana State University Museum of Natural Science. The project was approved by LSU's Institutional Animal Care and Use Committee (\#16-039).

\section{REFERENCES}

Bangs, O.C. \& Peters, J.L. 1927. Birds from Maratua Island off the east coast of Borneo. Occasional Papers of Boston Society of Natural History, 5: 235-242.

Becking, L. E., Renema, W., Santodomingo, N.K., Hoeksema, B.W., Tuti, Y. \& de Voogd, N.J. 2011. Recently discovered landlocked basins in Indonesia reveal high habitat diversity in anchialine systems. Hydrobiologia, 677: 89-105.

Bergin, D., Chng, S.C., Eaton, J.A. \& Shepherd, C.R. 2017. The final straw? An overview of Strawheaded Bulbul Pycnonotus zeylanicus trade in Indonesia. Bird Conservation International, $1-7$.

Chua, V.L., Phillipps, Q., Chuan Lim, H., Taylor, S.S., Gawin, D.F., Rahman, M.A., Moyle, R.G. \& Sheldon, F.H. 2015. Phylogeography of three endemic birds of Maratua Island, a potential archive of Bornean biogeography. Raffles Bulletin of Zoology, 63: 259-269.

Clements, J.F., Schulenberg, T.S., Iliff, M.J., Roberson, D., Fredericks, T.A., Sullivan, B.L. \& Wood, C.L. 2017. The eBird/Clements checklist of birds of the world: v2017. http:// www.birds.cornell.edu/clementschecklist/download.

de Bruyn, M., Stelbrink, B., Morley, R.J., Hall, R., Carvalho, G.R., Cannon, C.H., van den Bergh, G., Meijaard, E., Metcalfe, I., Boitani, L., Maiorano, L., Shoup, R. \& von Rintelen, K. 2014. Borneo and Indochina are major evolutionary hotspots for Southeast Asian biodiversity. Systematic Biology, 63: 879-901. 
de Voogd, N.J., Becking, L.E. \& Cleary, D.F.R. 2009. Sponge community composition in the Derawan islands, NE Kalimantan, Indonesia. Marine Ecology Progress Series, 396:169-180.

Dickinson, E. \& Dekker, R. 2002. Systematic notes on Asian birds. A preliminary review of the Pycnonotidae. Zoologische Verhandelingen, 25: 93-114.

Eaton, J.A., Shepherd, C.R., Rheindt, F.E., Harris, J.B.C., van Balen, S., Wilcove, D.S. \& Collar, N.J. 2015. Trade-driven extinctions and near-extinctions of avian taxa in Sundaic Indonesia. Forktail, 1-12.

Eaton, J.A., van Balen, B., Brickle, N.W. \& Rheindt, F.E. 2016. Birds of the Indonesian Archipelago, Greater Sundas, and Wallacea. Barcelona: Lynx Edicions.

Fooden, J. 1995. Systematic review of Southeast Asian longtail macaques, Macaca fascicularis (Raffles, 1821). Fieldiana Zoology, 81: 1-206.

Hoogerwerf, A., 1962. Notes on Indonesian birds with special reference to the avifauna of Java and the surrounding small islands (I, II, III). Treubia, 26: 11-291.

Hoogerwerf, A., 1965. Pycnonotus plumosus subspp. with the description of a new subspecies from Bawean island. Bulletin of the British Ornithologists' Club, 85: 47-53.

Hoogerwerf, A., 1966. Notes on the islands of Bawean (Java Sea) with special reference to the birds (I). The Natural History Bulletin of the Siam Society, 21: 313-340.

Hoogerwerf, A., 1967. Notes on the islands of Bawean (Java Sea) with special reference to the birds (II). The Natural History Bulletin of the Siam Society, 22: 15-103.

Jepson, P. \& Ladle, R.J. 2005. Bird-keeping in Indonesia: Conservation impacts and the potential for substitution-based conservation responses. Oryx, 39: 442-448.

Kier, G., Kreft, H., Lee, T.M., Jetz, W., Ibisch, P.L., Nowicki, C., Mutke, J. \& Barthlott, W. 2009. A global assessment of endemism and species richness across island and mainland regions. Proceedings of the National Academy of Sciences, 106: 9322-9327.

Lim, H.C., Gawin, D.F., Shakya, S.B., Harvey, M.G., Rahman, M.A. \& Sheldon, F.H. 2017. Sundaland's east-west rain forest population structure: variable manifestations in four polytypic bird species examined using RAD-Seq and plumage analyses. Journal of Biogeography, 44: 2259-2271.

MacArthur, R.H., Diamond, J.M. \& Karr, J.R. 1972. Density compensation in island faunas. Ecology, 53: $330-342$.

Manthey, J.D., Moyle, R.G., Gawin, D.F., Rahman, M.A., Ramji, M.F.S. \& Sheldon, F.H. 2017. Genomic phylogeography of the endemic Mountain Black-eye of Borneo (Chlorocharis emiliae): montane and lowland populations differ in patterns of Pleistocene diversification. Journal of Biogeography, 44: 2272-2283.

Ng, P.K.L. \& Tomascik, T. 1994. Orcovita saltatrix, a new genus and species of anchialine varunine crab (Crustacea: Decapoda: Brachyura: Grapsidae) from Kakaban Island, Indonesia. Raffles Bulletin of Zoology, 42: 937-948.

Nijman, V., 2004. Survey of birds of prey and owls (Falconiformes and Strigiformes) on Bawean, Java Sea, with records of three species new to the island. Raffles Bulletin of Zoology, 52: 647-651.

Nijman, V., 2006. The endemic Bawean Serpent-eagle Spilornis baweanus: habitat use, abundance and conservation. Bird Conservation International, 16: 131-143.

Oberholser, H.C., 1917. The birds of Bawean Island, Java Sea. Proceedings U.S. National Museum, 52: $183-198$.

Phillipps, Q. \& Phillipps, K. 2014. Phillipps' Field Guide to the Birds of Borneo. Third Edition. Oxford: John Beaufoy.

Riley, J.H. 1930. Birds from the small islands off the northeast coast of Dutch Borneo. Proceedings of the United States National Museum, 77 (2835):1-23. 
Shakya, S.B., Haryoko, T., Burner, R.C., Prawiradilaga, D.M. \& Sheldon, F.H. 2018. Preliminary assessment of community composition and phylogeographic relationships of the birds of the Meratus Mountains, south-east Borneo, Indonesia. Bulletin of the British Ornithologists' Club, 138: 45-66.

Shakya, S.B. \& Sheldon, F.H. 2017. The phylogeny of the world's bulbuls (Pycnonotidae) inferred using a supermatrix approach. Ibis, 159: 498-509.

Sheldon, F.H., Lim, H.C. \& Moyle, R.G. 2015. Return to the Malay Archipelago: The biogeography of Sundaic rainforest birds. Journal of Ornithology, 156: 91-113.

Sheldon, F.H., Lohman, D.J., Lim, H.C., Zou, F., Goodman, S.M., Prawiradilaga, D.M., Winker, K., Braile, T.M. \& Moyle, R.G. 2009. Phylogeography of the magpie-robin species complex (Aves: Turdidae: Copsychus) reveals a Philippine species, an interesting isolating barrier, and unusual dispersal patterns in the Indian Ocean and Southeast Asia. Journal of Biogeography, 36: 1070-1083.

Shepherd, C.R., Eaton, J.A. \& Chng, S.C. 2016. Nothing to laugh about-the ongoing illegal trade in laughing thrushes (Garrulax species) in the bird markets of Java, Indonesia. Bird Conservation International, 26: 524-530.

Vorderman, A.G. 1892. Bijdrage tot de kennis der avi fauna van het eiland Bawean. Natuurkundig Tijdschrift voor Nederlandsch, 51: 417-422.

Voris, H.K., 2000. Maps of Pleistocene sea levels in Southeast Asia: shorelines, river systems and time durations. Journal of Biogeography, 27: 1153-1167.

Wilcove, D.S., Giam, X., Edwards, D.P., Fisher, B. \& Koh, L.P. 2013. Navjot's nightmare revisited: logging, agriculture, and biodiversity in Southeast Asia. Trends in Ecology and Evolution, 28: $531-540$. 